\title{
Employing Virtual Reality in Academia
}

\author{
Matthew Montebello and Vanessa Camilleri
}

\begin{abstract}
The importance of the continuous development and education of every professional cannot be authentically reinforced enough in the case of academics as they are the source of knowledge, inspiration and leadership to tomorrow's professionals. The use of innovative technologies and pedagogies assist in enabling and facilitating the learning process especially in specific circumstances where the learners are educators and the domain is their area of specialization. In this article, we make the case for the pedagogic role of Virtual Reality techniques as part of the Career Professional Development of educators and academics in general. We discuss issues surrounding this concept and present our recommendations, the foreseeable future of this particular application of virtual reality, and draw our conclusions.
\end{abstract}

Index Terms-Academic training, continuous professional development, CPD, virtual reality, VR.

\section{INTRODUCTION}

The necessity and indispensability of maintaining up-to-date with the most recent developments, techniques and methodologies cannot be emphasized enough for a professional person. One distinctive characteristics of a true professional is a continuous commitment to lifelong and in-service training to ensure their acquired skills and accumulated knowledge remains sharp and in-line with the state-of-the-art. Continuous Professional Development (CPD) courses and initiatives are one of the most common and effective methodologies of how professional people endeavor to pursue their career in the best way possible while pledging to keep on expertly providing what they have been schooled and disciplined to carry out to the benefit of a civilized society that seeks and remunerates their services. Their domain of expertise evolves over time, as do the methods employed, processes followed, and new knowledge that enriches the same domain. The professional is incessantly obliged to ensue any of the potential CPD avenues available in an attempt to seek optimal career opportunities and flawless performance.

CPD traditionally refers to purposely planned and designed courses that are normally delivered to a group of professionals from the same field with the educational objective to fill the gap that over the months and years inevitably developed. The problem with this approach is that different peers within the same profession have different needs and requirements and thereby might require a distinctive and tailored procedure for them to fruitfully

Manuscript received October 30, 2017; revised May 11, 2018.

M. Montebello is with the AI Department, the ICT Faculty, the University of Malta. He is also with the College of Education, the University of Illinois, Urbana-Champaign, USA (e-mail: matthew.montebello@um.edu.mt).

V. Camilleri is with the AI Department, the ICT Faculty, the University of Malta, USA (e-mail: vanessa.camilleri@um.edu.mt). benefit from a CPD process. The exception to this is solely when the same unvaried information or training is required to be conveyed and invariably delivered to all the professionals in that particular field. This will not constitute all the necessary professional development required to these experts as each individual person still requires focused training and acquisition of specific supplement knowledge. The career needs and educational objectives of two professionals from within the same area of expertise potentially and indisputably require a differentiated CPD process or technique that optimally maintains their respective professional status. Some professionals prefer to adopt a daily or weekly routine making good use of available resources online or through subscription of domain journals and other publications. Other opt to follow self-directed courses or MOOCs that address their specific needs. While others just entrust their professional needs through regular and routinely CPD courses they subscribe to without having the burden to continuously apprehensively ponder if they are doing enough or the right thing. A healthy mixture of these techniques is also possible and realistic, in fact a number of researchers [1]-[3] conclude that a blended fusion of CPD approaches is more effective and potentially encompass the heterogeneous needs of each individual professional. Bosschieter highlights a number of methodologies available to support all professionals to reach their CPD goals. Apart from the traditional CPD courses, the author argues in favour of freely available software, apps, webinars and other web-based and multi-media learning methods that one needs to identify, access and take advantage of. These alternate CPD approaches cater for different professional domains and can also assist in supplementing the information and skills attained from formal courses. Technology and the use of new and alternate media have undoubtedly facilitated the relevance and applicability of such methodologies to assist experts in their pursuit to maintain the highest level of professionalism in their domain of expertise.

Academics and education professionals have unique CPD needs, as all other domains, that go across a number of areas that all need to be taken into consideration to ensure a complete and successful development of any expert. Apart from the content and objectives of each CPD performed, other issues need to be considered that in some way or another influence the same process, including the pedagogy employed, the medium of delivery employed, as well as the provider or contributor. All these issues will be tackled in this article.

The rest of the article is organized as follows. In the next section, we will pursue where we left in the Introduction and analyze and investigate the specific CPD for academics. Section III will delve into the use of Virtual Reality (VR) as employed within educational environments and settings. The 
focus of this article is then presented in the next section as a model that embraces VR for academics' CPD is presented and justified. The future of this technology and our recommendations are also presented in this section before we draw our conclusions in the final section.

\section{CPD AND THE TEACHING PROFESSION}

In this section a thorough analysis of all the issues that affect the teaching profession will be done in the light of the necessary continuous development that an educator needs to be exposed to. It is not related to career progression requirements or any non-academic related matter. If an issue has any kind of influence on the educator when perform any teaching related task then it forms of the academic profession of that person and thereby the CPD matter discussed will apply.

A number of variable factors that necessitate an academic to pursue professional development practices have been identified by Day and Leitch [4] in a study to investigate the effectivess of CPD practices. They state that five dimensions of teacher development needs to be reflected in their CPD efforts. Classroom knowledge is the first dimension which brings up purely academic issues related to the actual practices in the classroom, and potentially include the subject matter but also cover pedagogies employed, teaching aids, assessment methods and syllabuses. CPD in such cases tend to be short and isolated training focussed on the specific novel knoweldge, practice or tool that is being introduced. However, some research [5], [6] has shown that a continuous CPD training is more effective than brief intensive one. A second dimension involves the academic role within the educational process whereby the teacher might be leading a topical area or performing a specific role. In either case further training or skills to adjust to the new role are required. Opfer and Pedder [7] point out that the role of professional educators is continuously changing due to a continuously changing environment around them, and thereby require constant adjusting and acclimatization. One such environment is the school and its policies which is considered another dimension to be taken into consideration when planning CPD activities for educators. Any activities outside the classroom that influence what transpires within the classroom has an effect on the academic performance of the same educator. These potentially could include evolving school policies, new initiatives or suspension of established practices. The fourth dimension is similar to the previous one but rather than the school itself as an external force to the classroom, other forces external to school that still influence the academic activities within the classroom. Some examples include novel curricula, alternate assessment methodologies, and externally funded resources. Finally, the academic's personal development constitutes the fifth and most important dimension. The extent that a CPD activity is successful depends on this dimension as the educator will evaluate such an experience from a personal point of view. In fact numerous research studies [7], [8] conclude that the main influences that determine the effectiveness of the CPD actvity depend on the educator or the activities in which the educator participates in. What emerges from these five dimensions is that the main issues why CPD activities for academics are instrumental depend on the academic as a person, the system around the academic, and finally the learners. The last two purposes are continuously shifting and transforming the entire environment around the educator who is compelled to adjust, re-train, and develop further. Day and Leitch [4] use these three purposes to interpret the overall effectiveness of CPD activities. The educator's overall performance including the rapport with individual students and other personal skills like creativity, enthusiasm, resourcefulness, proactive and ambition are considered as an important element that reflect the extent of a professional educator. The authors continue by highlighting that the extent of how much this rubs off onto the learners is also a reflection of an educator who is performing a professional job. The pupil's academic outcome is also a reflection of the educator and the extent of how much the educator provides an environment that is inductive to creativity, open thinking, opportunities and accessibility. The third purpose why academics require CPD is the school itself as it brings the previous two together within its settings. The different forces acting on the school render it into a fluctuating entity that the educator and the learner have to deal with. School policies, assessment standards, logistics, and administrative issues are all potential dynamics that will eventually have their ripple effect on all the educators and their profession.

The focus of this article is on the educator as a professional and the necessary career needs to ensure optimal service rendered, in particular focusing on educators in higher education. Academics form part of the educators' profession and are subject to all the forces and issues discussed above. However, their area of expertise is not only much more finely focused and at a greater depth than kindergarten, primary, secondary, and post-secondary teachers, they have also been contributing to the body of knowledge within their particular domain. This implies that the need for them to be conversant, cognizant, and fluent with the state-of-the-art of their areas of expertise, is even greater and indispensable. Botham [9] investigates the specificity of CPD for academic within higher education institutions in UK and concludes that apart from the fact that both the academic and the institution benefit from such an exercise, further contributions to the knowledge domain may result, as a result of which they enrich their students' educational exposure and university experience. Other higher education researchers [10], [11] have also linked enhanced university students experience and their respective lecturer who was exposed to CPD. Other noteworthy research findings [9] indicate that academics who have gone through continuous CPD tend to be positively influenced in engaging and proactive in their area of expertise thereby enriching even more their own expertise and to their respective research community.

The use of technology in CPD activities has already been highlighter earlier in the introduction as Bosschieter [3] specifically states that the use of 'new media' (p. 71) has become increasingly central and pivotal in a predominantly technological society. The accessibility of resources online through a seamlessly connected lifestyle has enabled all levels of educators and trainers from schools, colleges, universities and industry to practice their CPD needs at 
anytime and anywhere. The availability of smart devices coupled with purposely-developed applications has enabled and facilitated the way educators ensure remain ahead of their game. Universities around the world [12] not online provide CPD courses for their academics but also provide the resources for them to access the plethora of available media in their own time and space. The favorable and constructive use of these available tools and applications requires a working knowledge and a comprehensive understanding of how to take full advantage of them, otherwise academics will not employ them if they do not envisage the benefits extracted [13].

\section{VR IN EDUCATION}

The new media, online resources and the ease of accessibility have opened up numerous ways of how academics can ensure to maintain their professional status and delivery. One such technology that has been widely employed in education [14]-[17] is Virtual Reality (VR) due to its active and immersive experience qualities that engages learners immediately without any possible distractions. Fernandez [18] argues on how VR improves the educational process as he concludes that such techniques require a holistic plan rather than employed in isolation. The author identifies six interlocking tasks that facilitate the academic adoption of VR in general, first of which is to train the educators as part of their career development. The other five tasks include conceptualization of prototypes, working in teams, software development, academic consultation, and the actual VR production. Within an academic scenario VR exhibits its formidable educational attributes as it pedagogically enriches the process through a differentiated value [19]. VR allows a lot of flexibility to ensure a realistic immersion whereby learners are challenged in ways that before where not possible, specific to any scenario or educational objective. Falloon [20] points out that VR is able to engage learners "in the development of higher order thinking skills, such as interpreting, analyzing, evaluating, synthesizing and solving complex problems" (p.108). This is particularly significant when abstract concepts need to be conveyed one way or another. Curcio, et. al., [15] reiterate that VR is able to conceptually represent abstract notions that learners, who were not capable to comprehend, are now able to do so.

Numerous of case studies of the use of VR in education can be found in the literature [12], [15], [17], [18] and how educators and institutions took full academic advantage of the novel technology. Advantages and capabilites that other industries have already been positively exploiting to enhance a game, simulate a medical procedure, train truck drivers, visit museums or inaccessible places. In all these cases the learners were able to experience a convincingly lifelike and authentic event that Kroes, et al. [21] were able to study and draw inferences on treatement strategies for disorders related to stress and anxiety. The authors were able to track the learners' emotional stages to investigate their contextual role within an experimentally controlled VR environment on learners' emotions, memories and learning. Dalgarno and Lee [22] also highlight the potential of VR as an educational medium as they identify a number of learning affordances. According to the authors VR facilitates learning tasks that lead to enhanced spatial knowledge representation, as well as the affordance of increased learning levels through experiental occurrences through VR. Other affordances include an elevated motivational sense that engages learners even more, enrichment of contextual learning, and the proliferation of quality collaborations as part of the learning process. Numerous other examples of how VR has been applied within an education setting can be found in the literature [16], [17], [19], [23], [24] highlighting its suitability, applicability and flexibility to lend itself to alternate and wide-ranging education scenarios.

\section{VR FOR ACADEMICS'S CPD - A MODEL}

The transition of employing VR in education with school children to its similar application to further train or education teachers and academics is straight forward as the focus in the previous sections was on VR's attributive values as an superior learning medium. What needs to be modified is the content and the focus of the materials employed, which are not an issue either. We argued earlier that such a medium can easily and effortlessly accommodate different abilities, ages, and subject domains. The main reason why this is so is due to its primary characteristic of simulating real life scenario through real video. The immersive and realistic environment viewed and surrounding the educator places the same educator within a micro-reality that simulates the everyday experience in the class. Such research has already been tested and documented [23] whereby novel technologies that are employed as teaching aids have been presented to educators to familiarize themselves and experiment with the different tools within a safe explorative environment. Similarly, initiatives that inspired the researchers at the University at Buffalo in the state of New York include SimSchools [25] that assists teachers in training by simulating a classroom. Another initiative [26] that simulated a classroom was developed at the University of Central Florida in an attempt to enhance the performance of educators in class. Bambury [27] adopts the same techniques to display a full and realistic 360 degrees visual to academics as part of CPD training offered whereby high-defition cameras have been employed to create the seamless environment through a VR goggles. Similarly, other commercial companies [28] have realized the full potential of VR and are offering higher education institutions CPD for their academic staff. Scientific proof has also been documented by Lamb [29] whereby physiological properties recorded of teachers and students using VR were similar to those of the same subjects in real life scenarios.

The advantages and benefits of employing VR for academics' CPD significantly outweigh the disadvantages which mainly revolve around the financial capital required to set up VR center and develop the content. However, as the technology evolves further and content repositories flourish in size and diversity, such costs are compensated by the academic outcome generated. Furthermore, some companies [30], [31] are already looking ahead in an attempt to enhance evenmore the user experience. Functionality whereby an academic can interactively communicate with individual 
learners within the three-dimensional video as if moving within a real classroom. The production of such content requires multi-threaded filming with artificial intelligent sensing and language processing to close the gap further between a real experience and a VR-rendered session. Academics will be able to perform their professional development by accustoming themselves to stressful scenarios within artificially generated encounters.

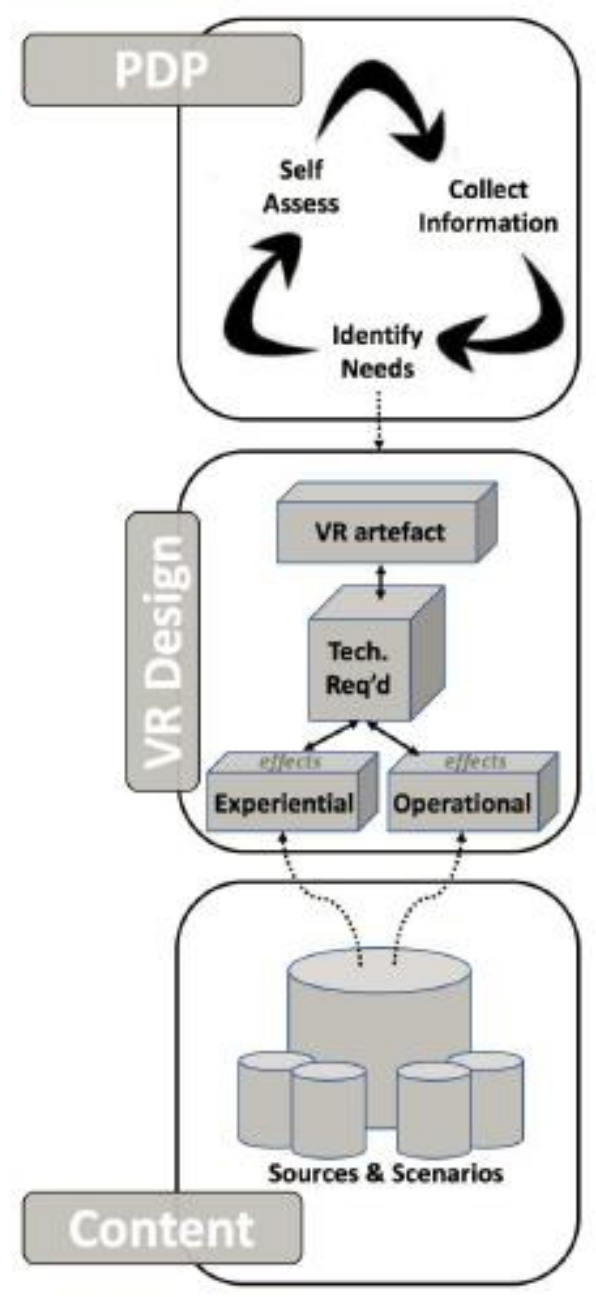

Fig. 1. VR conceptual model.

In an effort to ensure that every academic is presented by a tailored personal VR experience we devised a model whereby the career needs can be automatically identified and converted into VR content requirements. The model is necessary due to the independent nature of academics who do not have a subject coordinator similar to what secondary schools' teachers have, or a line-manager to identify the operational needs of workers. It is also necessary to have a model in place as some academics are not able to maintain their expertise up-to-date with the setback of falling behind within their profession and service provided. The model is based on the combination of the traditional personal development plan [32], [33] and a previously documented conceptual VR model [34]. Similar to the previous model our proposed model interfaces the combination of perceptual and muscle systems required to address academics' professional needs. These needs are identified through the career development plan and eventually materialized through the identification of contents and scenario necessary to generate the VR experience. Fig. 1 shows the combination of the three-tiered model to support the proposed model.

The professional development plan (PDP), in the top tier, is the first process required to trigger off the process. The PDP is a cyclic practice whereby academics are required to periodically follow a number of steps in an effort to assess, identify and fully consider any gaps in their professional persona. These could potentially include shortage of skills, undermined values, lack of interests, and a diluted research agenda, to mention just a few. Such an exercise requires an intense and profound self-assessment that some academics might require the use of a traditional personal development planner in order to comprehensibly encompass every aspect and detail. Once this exercise is performed and redone numerous times to re-assess and further refine the detailed aspects of one's professional status quo, an information collection exercise is required. This process depends very much so on the specific academic domain but still numerous discernable factors exist across different academics. Career progression and recognition within the area of expertise are obvious goals that every academic aspires to, as well as positive performance feedback from peers and students alike. Knowledge of top rated conferences and highly ranked journals give a clear indication of where the area of expertise is focusing and proceeding, thereby an excellent source of information to identify specific areas where the academic can excel and boost one's status. Following and maintaining updated information about the work and writings of the most influential experts and authorities within the specific area of research also gives insights and potential goals that an academic can aspire to and aim for. Similar to the self-assessment this is not a one-off exercise but a continuous and cyclic process or habit that the academic is required to perform as part of this conceptual model. The third part of this PDP that completes the cycle is to identify gaps and highlight specific needs. The compilation of the previous two tasks should explicitly uncover some needs, but the academic and the professional knowledge of the field of interest play an important role. Needs that include improvement of specific skills or competencies can be addressed through the need of specific and focused workshops and courses, while necessities that require greater exposure and a wider network might require identification of academic events and conferences that potentially require a physical presence but still require successful performances to access. This includes good practices in the areas of publications, project proposals, and research performances, that can be addressed by the VR model being proposed. This fulfills the function of the PRP with the three cyclic and recursive micro processes within it continuously active to further identify and refine the academic's needs to provide the required input to the second tire where the VR design is taken into consideration. Another input to the VR design is the third tier whereby the content is either stored or developed to provide the required source to populate and supply the necessary educational material. This could potentially be an academic repository of sources, learning materials as well as scenarios that VR producers can easily adopt and employ. The VR design within the second pier is adapted from Latta and Oberg [34] who proposed a methodology in how to isolate the human and technical 
aspects within a VR model. The authors, through the application of human perceptual and muscle systems, propose a VR system constructed on two views, namely, the human and the technical view as shown in Fig. 2. The human factor looks into what provides the stimulus for participants to act in the way they do from a physiological and psychological point of view. On the other hand, the environment factor is related to the technical details of the physical space itself together with all its attributes. These include functional properties provided within the environment together with the conceptual capabilities of the available tools and the possible practices that can be performed. The way that this model has been adapted to our proposal by defining what constitutes a VR system through their conceptual model. The VR artefact at the top tier of the design is equivalent to the computer-based interface to human perceptual and muscle systems. This final product represents the developed experience that brings together all the other steps within the proposed model. The required technologies to develop the VR artefact are represent in the middle level of the design. It represents an analysis of all the equipment and effects required to bring about the desired outcome. It is equivalent to the use of technology to create an environment that seems realistic within the Latta \& Oberg model [34]. Finally, at the bottom of the design are the desired effects, namely the experiential and operational. The experiential effects relate to the users' personal experiences while participating in the VR environment, while the operational effects relate to the operations performed while in the same environment. This completes our rationale behind each element within the proposed three-tier VR conceptual model that not only includes details on an academic's professional development plan, but also concepts behind a VR system from both the system's and academic's perspective.

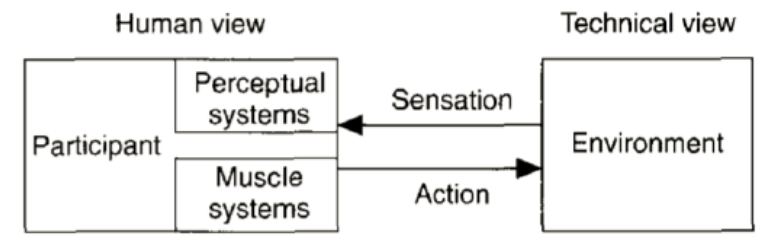

Fig. 2. VR System: Participant and environment [34].

\section{CONCLUSION}

In this article, we have argued about the importance and necessity of the continuous professional development of academics in an effort to ensure that provide a continuous and consistent educational service at the universities, as our society strongly depends on their knowledge, inspiration and skillful abilities to train and inspire tomorrow's leaders. We have reported how the use of innovative technologies and pedagogies play a major role in the pedagogical assistance to enable and facilitate the learning process. A stronger case was argued when the learners are not only educators themselves but academics at the pinnacle of every educational institution who require constant re-training in teaching skills but also in acquiring new knowledge and maintain fresh their previous skills and knowledge within their own specialized research area of interest. We then explained and justified the role and employment of virtual reality techniques and equipment within a learning environment to assist learners completely immerse themselves and barely distinguish between simulation and reality. Such virtual reality properties support and facilitate the learning process in general, but this article makes the case for the use of these techniques as part of the career professional development of academics. We do this by proposing a virtual reality conceptual model that brings together three important aspects in the realization of any VR artefact. The academic starts off by habitually practicing a periodic exercise involving continuous professional development planning, that will produce results that the virtual reality design aspect employs together with existent of newly developed resources.

\section{REFERENCES}

[1] K. Holloway, K. Arcus, and G. Orsborn, "Training needs analysis The essential first step for continuing professional development design," Nurse Education in Practice, vol. 28, pp. 7-12, 2018.

[2] K. Golnik, "Continuing professional development (CPD): Why should we continue to learn?" Community Eye Health Journal, vol. 30, no. 97, pp. 1-3, 2017.

[3] P. Bosschieter, "Continuing professional development (CPD) and the potential of new media," Indexer, vol. 34, no. 3, pp. 71-74, 2016.

[4] C. Day and R. Leitch, "The Continuing professional development of Teachers: Issues of coherence, cohesion and effectiveness," International Handbook of School Effectiveness and Improvement, Dordrecht, Springer, 2007, pp. 707-726.

[5] V. Collinson and T. Cook, "I d don't have enough time': Teachers' interpretations of time as a key to learning and school change," Journal of Educational Administration, vol. 39, no. 3, pp. 266-281, 2001.

[6] M. Garet, A. Porter, L. Desimone, B. Birman, and K. Yoon, "What makes professional development effective? Results from a national sample of teachers," American Educational Research Journal, vol. 38, no. 4, pp. 915-945, 2001.

[7] V. Opfer and D. Pedder, "The lost promise of teacher professional development in England," European Journal of Teacher Education, vol. 34, no. 1, pp. 3-24, 2011.

[8] R. M. McGill, "Professional development for teachers: how can we take it to the next level?," 2013.

[9] K. Botham, "The perceived impact on academicsâ€ TM teaching practice of engaging with a higher education institutionâ $\square{ }^{\mathbf{T M}_{S}}$ CPD scheme," Innovations in Education and Teaching International, pp. 1-12, 2017.

[10] A. Brew and P. Ginns, "The relationship between engagement in the scholarship of teaching and learning and students' course experience," Assessment and Evaluation in Higher Education, vol. 33, pp. 535-544, 2008.

[11] D. Parsons, I. Hill, J. Holland, and D. Willis, "Impact of teaching development programmes in higher education," HEA Research Series, 2012.

[12] J. Winter, R. Turner, L. Spowart, R. Muneer, and P. Kneale, "Evaluating academic development in the higher education sector: academic developers' reflections on using a toolkit resource," Higher Education Research \& Development, vol. 36, no. 7, pp. 1503-1514, 2017.

[13] R. Walker, J. Voce, J. Nicholls, E. Swift, J. Ahmed, S. Horrigan, and P. Vincent, "2014 Survey of Technology Enhanced Learning for higher education in the UK," UCISA, Oxford, 2014.

[14] B. Jones, "To raise teaching standards we must first improve the use of technology in the classroom," 2017.

[15] I. D. D. Curcio, A. Dipace, and A. Norlund, "Virtual realities and education," Research on Education and Media, vol. 8, no. 2, pp. 60-68, 2016.

[16] M. Hoffmann, T. Meisen, and S. Jeschke, "Shifting virtual reality education to the next level — Experiencing remote laboratories through mixed reality," presented at International Conference on Computer Science, Computer Engineering, and Education Technologies, Kuala Lumpur, 2014.

[17] H.-M. Huang, U. Rauch, and S.-S. Liaw, "Investigating learners' attitudes toward virtual reality learning environments: Based on a 
constructivist approach," Computers \& Education, vol. 5, pp. $1171-1182,2010$.

[18] M. Fernandez, "Augmented virtual reality: How to improve education systems," Higher Learning Research Communications, vol. 7, no. 1, pp. 1-15, 2017.

[19] C. Kumar, "A new frontier: How can you profit from augmented and virtual reality?" 2017.

[20] G. Falloon, "Using avatars and virtual environments in learning: What do they have to offer?" British Journal of Educational Technology, vol. 41 , no. 1 , pp. $108-122,2010$

[21] M. Kroes, J. Dunsmoor, W. Mackey, M. McClay, and E. Phelps, "Context conditioning in humans using commercially available immersive virtual reality," Scientific Reports, vol. 7, no. 1, 2017.

[22] B. Dalgarno and M. J. W. Lee, "What are the learning affordances of 3-D virtual environments?," British Journal of Educational Technology, vol. 41, no. 1, pp. 10-32, 2010.

[23] C. Anzalone, "UB's virtual reality expertise creates simulated classroom environment for aspiring teachers," 2017.

[24] M. Virvou and G. Katsionis, "On the usability and likeability of virtual reality games for education: The case of VR-ENGAGE," Computers \& Education, vol. 50, no. 1, pp. 154-178, 2008.

[25] M. Zibit and D. Gibson, "simSchool: The game of teaching," Innovate, vol. 1, no. 6, 2005.

[26] D. Ferrante, "Kickin' It New School," Pegasus, 2017.

[27] S. Bambury, "CPD in VR," 2017.

[28] L. Loewus, "How virtual reality is helping train new teachers," Education Week, vol. 37, no. 3, pp. 1-2, 2017.

[29] R. Lamb, "Virtual reality as a tool for engaging students," NatureResearch, 2017.

[30] Crosswater, "VR/360," 2017.

[31] Polygon, "The state of virtual reality," 2017.

[32] S. Beausaert, M. Segers, and W. Gijselaers, "The use of a personal development plan and the undertaking of learning activities, expertise-growth, flexibility and performance: the role of supporting assessment conditions," Human Resource Development International, vol. 14 , no. 5, pp. 527-543, 2011.

[33] C. Lejeune, D. Mercuri, S. Beausaert, and I. Raemdonck, "Personal development plans supporting employee learning and perceived performance: the moderating role of self-directedness," Human Resource Development International, vol. 19, no. 4, pp. 307-328, 2016.
[34] J. Latta and D. Oberg, "A conceptual virtual reality model," Computer Graphics and Applications, vol. 14, no. 1, pp. 23-29, 1994.

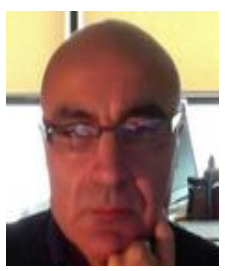

Matthew Montebello is an associate professor at the Department of Artificial Intelligence at the Faculty of ICT, University of Malta. He heads the Agent Technology Research Group at departmental level, as well as coordinates a number of Interest Groups within the same faculty. Before joining the University in 1999 with a $\mathrm{PhD}$ in Computer Science he was already heavily involved in Education in secondary schools after graduating in 1990 at the University of Malta B.Ed.(Hons) degree. Having obtained an extensive teaching experience and having been involved with the introduction of computer labs through the Ministry of Education, he proceeded to follow the Computer Science domain when he pursued his post-graduate studies obtaining a Masters and a Doctorate at the Cardiff University in Wales in 1996 and 1998 respectively. Furthermore in 2009 and 2016 he also completed an M.A. and an Ed.D. (Higher Education) specialising in the application of artificial intelligence to e-learning.

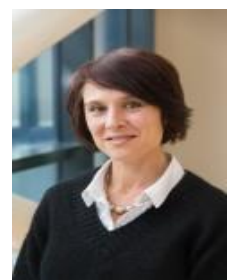

Vanessa Camilleri lectures at the Faculty of ICT. Her work is in the area of human-computer interaction, virtual reality applications, and serious games. Before she joined the Faculty of ICT she lectured at the Faculty of Education, where she was teaching Computing in Education, as well as Educational Technologies (including open education, and eLearning) for a number of years.

These experiences have contributed to Dr Camilleri's strong beliefs in inter-Faculty collaboration. She is also a great believer in quality Education that is a key factor for success in today's world, and that is further enhanced by the digital possibilities and technology applications that are driving much of what makes up our society. Her publications are mostly in the areas of autonomous learning projects and possibilities and her current research interests are in the field of Virtual Worlds for Education and Serious Applications. She is also involved in two funded projects that deal with the use of games for learning. Together with teaching, research constitutes a very important component in Dr. Camilleri's academic career. 